\title{
Kim Stroumza, Heinz Messmer (dir.), Langage et savoir-faire: des pratiques professionnelles du travail social et de la santé passées à la loupe
}

\section{Adrien Mathy}

revues.org

Édition électronique

URL : http://lectures.revues.org/21955

ISSN : 2116-5289

\section{Éditeur \\ Centre Max Weber}

Ce document vous est offert par Université de Liège

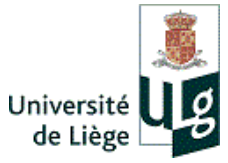

Référence électronique

Adrien Mathy, « Kim Stroumza, Heinz Messmer (dir.), Langage et savoir-faire : des pratiques professionnelles du travail social et de la santé passées à la loupe », Lectures [En ligne], Les comptes rendus, 2016, mis en ligne le 15 décembre 2016, consulté le 22 février 2017. URL : http:// lectures.revues.org/21955

Ce document a été généré automatiquement le 22 février 2017.

(c) Lectures - Toute reproduction interdite sans autorisation explicite de la rédaction / Any replication is submitted to the authorization of the editors 


\title{
Kim Stroumza, Heinz Messmer (dir.), Langage et savoir-faire: des pratiques professionnelles du travail social et de la santé passées à la loupe
}

\author{
Adrien Mathy
}

1 Premier opus de la collection «Le geste social » des éditions de la Haute école de travail social, ce recueil dirigé par Karim Stroumza et Heinz Messmer a pour ambition d'ouvrir l'ensemble des pratiques professionnelles aux postures critiques et réflexives. Les contributions ont pour dénominateur commun d'aborder l'ambivalente relation au langage qu'entretiennent les professionnels dans le champ du travail social et de la santé. En effet, afin de donner aux praticiens les outils nécessaires à une interrogation réflexive, la jeune collection genevoise réalise son souhait « d'ouvrir des espaces de réflexion sur les problèmes publics contemporains $»^{1}$. Elle met ainsi à disposition un lieu de débat, qui fait véritablement office d'interface entre postures analytiques ou théoriques et réalité concrète de la praxis sociale : les diverses contributions de ce premier numéro rendent compte de la pertinence et de la pérennité d'approches qui relèvent non seulement de la praxématique ${ }^{2}$, au sens large, mais surtout d'une praxéologie intégrant l'acte langagier dans la dynamique de l'action humaine.

2 Afin de traiter cette thématique complexe avec pertinence, les contributions opèrent un zoom sur un contexte d'action et de production particulier en ne prenant pas pour objet le langage, mais la relation au langage. En opérant ce focus, l'ouvrage donne la parole à des auteurs qui sont, certes, chercheurs, mais qui se définissent aussi comme formateurs, ancrant leur analyse dans une expérience réelle en appréhendant les productions langagières et discursives au sein de l'action professionnelle. Les analyses recueillies donnent donc une place importante à l'exemplification et l'expérience. Il est par ailleurs possible, malgré la diversité des analyses, d'isoler des traits méthodologiques saillants qui démontrent la cohérence des démarches répertoriées. L'intégralité des contributions 
rendent ainsi compte de la dynamique des discours qui se construisent dans et par l'interaction entre les individus. Chaque article prend soin de définir le rôle du locuteur dans l'interaction, selon qu'il est acteur, patient, bénéficiaire, etc. En effet, comme nous le comprenons à la lecture de l'ouvrage, tout je se construit en rapport avec un tu et son discours ne fait sens que dans le contexte de sa production, en l'occurrence le milieu du travail social et de la santé. Après quelques prolégomènes théoriques exposés par Kim Stroumza, l'ouvrage s'ouvre sur un article de Patrick Rousseau qui illustre parfaitement l'approche théorique exposée. En s'intéressant à la production écrite de professionnels dans un service d'action éducative en milieu ouvert (AEMO) judiciaire, l'auteur étudie une pratique discursive réalisée dans un contexte idiosyncrasique. Il confronte ainsi, au fil d'une analyse comparative, les productions des éducateurs qui sont restreintes à l'usage interne (rapport de réunion, entretien avec les parents ou les enfants, etc.) et celles qui sont adressées au juge. En étudiant les variations entre les deux types de productions, l'auteur met au jour une série de stratégies discursives (usage de l'euphémisme, effacement des marqueurs personnels, etc.) qui visent à maintenir l'équilibre paradoxal de la relation d'aide qui est caractérisée par l'obligation de contrôler la famille et de " protége[r] un enfant en danger » tout en le « [maintenant] dans sa famille, c'est-à-dire précisément là où il est le plus menacé » (p. 31).

3 Tandis que l'article de Rousseau étudie, à travers des productions écrites, la manière dont l'identité des éducateurs s'actualise en discours, les deux articles qui suivent s'inscrivent dans l'approche dite conversationnelle et s'intéressent à la construction de l'identité par le discours oral. Le premier, co-écrit par Heinz Messmer et Fabienne Rotzetter, propose un panorama détaillé des recherches sur la méthode EMCA (ethnomethodological conversation analysis). La place que l'EMCA accorde à la réflexivité et à l'auto-analyse justifie en effet pleinement l'intérêt de cet aperçu. Les auteurs appliquent leur méthode à une série d'entretiens entre assistants sociaux et bénéficiaires ${ }^{3}$ et proposent une perspective analytique originale. Messmer et Rotzetter remarquent qu'une aide ne peut être accordée au bénéficiaire que s'il est légitime dans sa demande. Il ressort de leur analyse que cette légitimité est construite par des interactions entre assistants sociaux et bénéficiaires qui s'apparentent à des processus de «clientification » (p. 61). Ces processus réalisent dans le langage la dialectique complexe entretenue entre les nécessités irréfragables de l'institution (qui doit rendre le bénéficiaire « aidable »), et « les logiques de l'interaction ordinaire » (p. 21).

4 Le second article, auquel ont contribué Esther González-Martínez, Vassiliki Markaki, et Fanny Bovey, s'inscrit dans la continuité du précédent en conceptualisant le langage comme " une élaboration commune et interactive » (p. 86). Les auteurs s'intéressent ainsi aux interactions entre une diététicienne et une infirmière, dans le cadre d'une procédure codifiée de demande de régime, qui sort des paradigmes conventionnels eu égard à son urgence. Procédant à l'analyse conversationnelles des échanges téléphoniques retranscrits, les auteurs formalisent la discussion en termes de séquence régie selon diverses configurations et révèlent les conditions selon lesquelles les intervenants coordonnent leur action. L'analyse montre comment le discours actualise «la collaboration interprofessionnelle et [le] partage des responsabilités » (p. 87) propres à une institution hospitalière.

5 Entérinant le fil rouge du discours construit de façon interindividuelle, les quatrième et cinquième articles s'intéressent respectivement à l'expérience $d u$ discours et à l'expérience dans le discours. La contribution d'Antoine Auchlin interroge, dans un propos 
particulièrement théorique relatif à l'incarnation du discours, les actualisations discursives de l'expérience du je. A priori sans rapport avec le thème de l'ouvrage, parce qu'il ne développe pas la question du travail social, l'article d'Auchlin est à propos car il questionne avec pertinence la possibilité d'analyser de façon objective une expérience aussi subjective que celle du discours. Il est en effet primordial, surtout dans une profession sociale, de construire une méthode d'analyse ad hoc qui échappe et à l'écueil objectiviste déshumanisant et prétendument "auto-suffisant»(p.117) et à l'écueil subjectiviste qui ne reconnaît pas la possibilité de généraliser et d'isoler certaines propriétés relatives à la subjectivité. C'est pourquoi Auchlin propose de s'intéresser au discours en tant qu'il est l'expérience d'un je et d'un tu qui se construisent mutuellement (comme l'ont illustré les articles précédents). Le discours n'est ni réduit à des propriétés linguistiques objectives ni restreint à l'expression subjective du je, il est une construction mutuelle et intersubjective qui n'a de sens que dans l'expérience de la conscience de soi qui émerge grâce audit discours.

6 La contribution de Nathalie Ilić s'intéresse quant à elle à «l'expérience en discours ». Poursuivant un travail de recherche encouragé par le CER ${ }^{4}$, l'auteur analyse les documents éthiques soumis aux patients qui se portent volontaires pour des essais cliniques. Le document est alors analysé comme une mise en discours écrite d'une expérience qui diverge fondamentalement selon qu'elle est relative au point de vue du médecin ou du patient. Les deux acteurs, compte tenu des obligations de l'institution, doivent néanmoins passer par cette médiation hybride et asymétrique. Le patient qui ne peut comprendre l'intégralité du protocole (car écrit dans l'idiolecte de l'expert médical) et qui doit s'en remettre en partie au hasard (le traitement étant expérimental) ne jouit pas de la même position que l'investigateur qui réifie le corps du patient, devenu à la fois sujet puisqu'il donne son accord et objet puisque par cet accord il accepte de devenir objet de l'expérimentation.

7 Les deux derniers articles, respectivement de Kim Stroumza et de Roland Becker-Lenz ferment l'ouvrage sur une perspective plus théorique. S'inscrivant dans le champ de la pragmatique intégrée, en assumant une filiation avec Auchlin, Stroumza étudie les rapports de pouvoir que le langage réalise. En auscultant l'interaction informelle entre une patiente en situation d'handicap psychiatrique et une éducatrice du foyer, Stroumza met en lumière les façons dont le langage construit, relationnellement, l'identité des deux intervenants, ainsi que les conditions selon lesquelles il est possible d'équilibrer deux référentiels a priori inconciliables - celui de l'amour-vocation et celui de la professiontechnicité. Il entérine le propos d'Auchlin en concluant qu'il existe une conceptualisation du langage qui diffère de la lecture subjectiviste et de la lecture objectiviste.

8 L'article de Becker-Lenz conclut enfin l'ouvrage sur une approche quasi absente du domaine francophone, malgré sa grande présence en Allemagne, à savoir l'herméneutique objective. Après un état de l'art exhaustif, l'auteur expose quelques desideratas relatifs aux méthodes d'application concrète et aux avantages de celles-ci dans le cadre du diagnostic social (c'est-à-dire la définition objective ou consensuelle des problèmes des usagers). Becker-Lenz s'attache à rendre concret, notamment par des métaphores explicatives, une approche a priori très abstraite. Dans cette optique, il fournit aux praticiens une technique d'analyse formalisée qui permet d'encadrer les débats et les prises de décision, tout en évitant les choix arbitraires. Cette perspective presque didactique est, somme toute, nécessaire au lecteur. 
9 Parce qu'il s'adresse autant à des professionnels qu'à des étudiants ou des chercheurs, l'ouvrage réalise un équilibre complexe entre des propos théorétiques parfois abscons et une exigence de l'exemplification couplée à un désir éducatif parfaitement illustré par cette dernière contribution.

\section{NOTES}

1. Voir la ligne éditoriale de la revue sur http://www.lcdpu.fr/collections/legestesocial/ (consulté le 21/11/2016).

2. Il nous semble pertinent de rattacher les diverses contributions à la praxématique qui étudie comment un discours produit du sens dans un contexte donné.

3. Terme usuellement utilisé, par analogie au couple patient/médecin, pour désigner les individus recevant une aide de l'institution sociale. La particularité de l'article en question est d'y substituer le terme de client afin de répondre à une problématique particulière.

4. Le CER (commission d'éthique à la recherche) des Hôpitaux universitaires genevois est à l'origine d'un premier protocole de recherche déposé en 2009 sur lequel Ilić donne quelques éléments explicatifs.

\section{AUTHOR}

\section{ADRIEN MATHY}

